\title{
Cannabidiol attenuates cognitive deficits and neuroinflammation induced by early alcohol exposure in a mice model
}

\author{
Alba García-Baos ${ }^{\text {a }}$, Xavier Puig-Reyne ${ }^{\text {a }}$, O.Óscar García-Algar ${ }^{c}$, Olga Valverde ${ }^{\text {a,b, * }}$ \\ ${ }^{a}$ Neurobiology of Behaviour Research Group (GReNeC-NeuroBio), Department of Experimental and Health Sciences, Universitat Pompeu Fabra, Barcelona, Spain \\ ${ }^{\mathrm{b}}$ Neuroscience Research Programme, IMIM-Hospital del Mar Research Institute, Barcelona, Spain

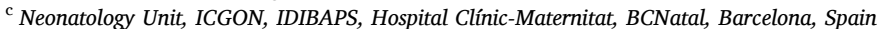

\section{A R T I C L E I N F O}

\section{Keywords:}

Foetal alcohol spectrum disorder

Prenatal alcohol exposure

Cognitive deficits

Cannabidiol

Neuroinflammation

Chemical compounds studied in this article:

Cannabidiol (PubChem CID: 644019)

Ethanol (PubChem CID: 702)

\begin{abstract}
A B S T R A C T
Foetal alcohol spectrum disorder (FASD) is the umbrella term used to describe the physical and mental disabilities induced by alcohol exposure during development. Early alcohol exposure induces cognitive impairments resulting from damage to the central nervous system (CNS). The neuroinflammatory response accompanied by neurodegenerative mechanisms contribute to those detrimental alterations. Cannabidiol (CBD) has recently emerged as an anti-inflammatory drug that might be useful to treat several neuropsychiatric disorders. In our study, we assessed the effects of CBD on long-lasting cognitive deficits induced by early alcohol exposure. Furthermore, we analysed long-term pro-inflammatory and apoptotic markers within the prefrontal cortex and hippocampus. To model alcohol binge drinking during gestational and lactation periods, we used pregnant C57BL/ 6 female mice with time-limited access to $20 \% \mathrm{v} / \mathrm{v}$ alcohol solution. Following the prenatal and lactation alcohol exposure (PLAE), we treated the male and female offspring with CBD from post-natal day (PD) 25 until PD34, and we evaluated their cognitive performance at PD60. Our results showed that CBD treatment during peri-adolescence period ameliorates cognitive deficits observed in our FASD-like mouse model, without sex differences. Moreover, CBD restores the PLAE-induced increased levels of TNF $\alpha$ and IL- 6 in the hippocampus. Thus, our study provides new insights for CBD as a therapeutic agent to counteract cognitive impairments and neuroinflammation caused by early alcohol exposure.
\end{abstract}

\section{Introduction}

Alcohol is alarmingly used by pregnant women nowadays, and approximately one third of those who reported alcohol consumption over pregnancy engaged in binge drinking [1], which is particularly harmful to the brain of a developing foetus [2]. FASD comprises a wide range of morphological and functional anomalies that arise from early alcohol exposure. The consequences of alcohol drinking during pregnancy and/or breastfeeding may vary in severity across the spectrum [3]. However, cognitive impairments are core symptoms among FASD patients, especially learning and memory deficits which are highly incident in all diagnoses [4].
Since FASD is a neurodevelopmental disorder, an early intervention is key to minimise the devastating effects of alcohol exposure. Despite the lack of curative treatment, miscellaneous interventions have been employed to ameliorate their quality of life, such as parental training, behavioural education, and pharmacotherapy. Thereby, antiinflammatory agents have been proposed as treatment for FASD to address the neuroinflammatory phenotype observed in such patients $[5$, 6]. In fact, previous studies showed that prenatal alcohol exposure induces microglial activation as well as increased production of cytokines in the foetal rodent brain [7-9], which might persist until the adulthood [10]. Importantly, earlier preclinical research indicated that deficiency of toll-like receptor 4 (TLR4), a receptor involved in the activation of

Abbreviations: CBD, cannabidiol; CNS, central nervous system; COX, cyclooxygenase; CB1R, cannabinoid receptor type 1; CB2R, cannabinoid receptor type 2; DID, drinking in the dark; FAAH, fatty acid amide hydrolase enzyme; FASD, Foetal alcohol spectrum disorder; HPC, hippocampus; IL, interleukin; NF-kB, nuclear factor Kappa-B; NLRP, NOD-like receptor protein; NOL, novel object location; NOR, novel object recognition; PD, post-natal day; PFC, prefrontal cortex; PLAE, prenatal and lactation alcohol exposure; PPAR, peroxisome proliferator-activated receptor; TNF $\alpha$, tumour necrosis factor alpha; TLR4, toll-like receptor 4; VEH, vehicle.

* Correspondence to: Neurobiology of Behaviour Research Group (GReNeC - NeuroBio), Department of Experimental and Health Sciences, Universitat Pompeu Fabra, Dr. Aiguader 88, Barcelona 08003, Spain.

E-mail address: olga.valverde@upf.edu (O. Valverde). 
innate immune response [11], prevents the pro-inflammatory response along with long-term behavioural impairments found in alcohol exposed offspring [12]. In addition, the neuroinflammatory and apoptotic signalling in cerebral cortex and hippocampus can be blocked by the anti-inflammatory agent resveratrol, preventing cognitive deficits in early alcohol exposed rats [13]. Altogether, these findings support that the neuroinflammatory state induced by developmental alcohol exposure is a relevant trigger for long-term cognitive dysfunctions.

In the last decades, studies have shown that some cannabinoids have a neuroprotective pharmacological profile through multiple mechanisms [14]. These compounds exhibit a potential therapeutic role in the context of neuroinflammatory and neurodegenerative disorders, including the alcohol-induced neuroinflammation [15]. Among phytocannabinoids, cannabidiol (CBD) is able to interact with multiple targets within the central nervous system (CNS) exerting distinct pharmacological effects [14]. For instance, CBD increases the levels of endocannabinoids as consequence of fatty acid amide hydrolase enzyme (FAAH) inhibition [16]. The increased tone of endocannabinoids decreases neuronal damage, which is prevented by cannabinoid receptor type 1 (CB1R) and cannabinoid receptor type 2 (CB2R) antagonisms [17]. CBD can activate non-cannabinoid receptor pathways to exert its neuroprotective effects as well [18]. Previous studies showed that CBD can reduce microglial activation and pro-inflammatory cytokine production $[19,20]$. Although the mechanisms underlying its anti-inflammatory effects are not entirely understood, the activation of peroxisome proliferator-activated receptor (PPAR) gamma [21,22] could play a key role. Other receptors, such as transient receptor potential vanilloid $1[23,24]$ or $\mathrm{G}$ protein-coupled receptor 55 , might also be involved in these responses [18].

Additionally, CBD might be a promising therapeutic candidate for the management of sequels induced by excessive alcohol exposure. Preclinical data revealed that CBD attenuates the neurodegeneration within hippocampus [25] and entorhinal cortex [25,26] caused by a rodent model of binge-like alcohol drinking. Notwithstanding, CBD effect on developmental alcohol exposure has never been addressed.

To evaluate the impact of alcohol exposure during early developmental periods in the offspring, we have developed a reliable mouse model of binge-like alcohol drinking during gestational and lactation periods. Our previous findings indicated that early alcohol-exposed mice show spatial, working and recognition memory impairments [10,27], which mimics the behavioural impairments found in FASD patients. In the present study, we aimed to explore the therapeutic effects of CBD demonstrating that the phytocannabinoid might attenuate cognitive deficits induced by developmental alcohol exposure, conceivably through an anti-inflammatory mechanism.

\section{Materials and methods}

\subsection{Animals}

Male and female C57BL/6 were purchased from Charles River (Barcelona, Spain) and transported to our animal facility (UBIOMEX, PRBB) to be used as breeders. Animals were housed in a room with controlled temperature- $\left(21 \pm 1{ }^{\circ} \mathrm{C}\right)$, humidity- $(55 \pm 10 \%)$ and lighting (light remained on between 7:30 p.m. and 7:30 a.m.). Mice were allowed to acclimatise to the new environmental conditions for at least 1 week prior to experimentation, which occurred during the dark phase under a dim red light. Animals were 12 weeks old when the breeding began, and they were housed 1 male with 2 females. After successful mating, pregnant females were individualised and observed daily for parturition. For each litter, the date of birth was designated as PD0. Pups remained with their mothers for 21 days and were then weaned (PD21). After weaning, male and female offspring were housed in separated groups. Food and water were available ad libitum, except when water was substituted by alcohol to carry out the drinking in the dark (DID) test. All animal care and experimental procedures were conducted in accordance with the European Union Directive 2010/63/EU regulating animal research and were approved by the local Animal Ethics Committee (CEEA-PRBB).

\subsection{DID test}

This procedure was conducted as previously reported [10,27,28], which allows to model an early alcohol exposure under a binge-like drinking pattern. Briefly, two days after mating, pregnant females were randomly assigned to two groups: alcohol or water (control). Three hours after the lights were turned off, the water bottles were replaced with $10-\mathrm{ml}$ graduated cylinders fitted with sipper tubes containing either $20 \%$ (v/v) alcohol in tap water or only tap water. From Monday to Wednesday (days 1, 2, 3), pregnant females were allowed to voluntarily drink for $2 \mathrm{~h}$-access period. On Thursday (day 4), the drinking-access period was extended to $4 \mathrm{~h}$. Volumes consumed were recorded just after the drinking-access periods. During this procedure, all the females were individually housed in order to record individual fluid intakes. Following the drinking-access period, water bottles were returned to the home cage. Fluid intakes ( $\mathrm{g} / \mathrm{kg}$ of body weight) were calculated for each day on the basis of average 2-day body weight values, since dams were weighed at 2-day intervals (Monday and Wednesday).

In our experiment, DID test consisted of six consecutive weeks, encompassing prenatal and lactation periods. Therefore, each pregnant dam was exposed to six binge-like drinking sessions (day 4), following the habituation period (days 1,2,3). As previously reported, blood alcohol concentration of dams reached levels of $\sim 0.8 \mathrm{~g} / \mathrm{L}$ after the last binge-like drinking session [10], which is in accordance with "Drinking Levels Defined" reported by NIAAA [29].

\subsection{Drugs}

Ethyl alcohol was purchased from Merck Chemicals (Darmstadt, Germany) and diluted in tap water to obtain a $20 \%$ (v/v) alcohol solution. CBD (20 mg/kg i.p.) was generously provided by Phytoplant Research S.L., (Córdoba, Spain). The dose of $20 \mathrm{mg} / \mathrm{kg}$ was chosen based on other studies showing this dose within the anti-inflammatory therapeutic range in rodents and humans [30]. CBD was first mixed with $2 \%$ Tween- 80 and, after being homogenised by grinding in a mortar, $0.9 \%$ $\mathrm{NaCl}$ was added.

\subsection{Experimental design}

Offspring mice were administered with vehicle or CBD $(20 \mathrm{mg} / \mathrm{kg}$ i. p.) during 10 consecutive days from PD25 until PD34, corresponding to the peri-adolescence period. The treatments were randomly assigned to either exposed to water (control) or to alcohol (PLAE group). Then, animals were left undisturbed at the home cage until reaching the adulthood (PD60), when the behavioural tests were carried out as shown in Fig. 1A. Male and female offspring were used as a whole population since there were not differences due to the sex factor. All groups contained balanced number of male and female to avoid this possible bias. At least eight litters were used for each experimental group and treatments were randomly distributed through all litters to avoid the litter effect.

\subsection{Reference memory test}

A black Y-maze (two equal $395 \mathrm{~mm}$-long arms, separated by $120^{\circ}$ angles) was employed to assess spatial reference memory [31]. This experiment consisted of two phases (training and testing session) with $1 \mathrm{~h}$ of inter-trial interval, which is considered as short-term spatial memory. In the training session, one arm of the Y-maze was closed off, which was designated as the novel arm. Then, the mouse was placed into the start arm of the maze and was allowed to freely explore the two remaining arms for $5 \mathrm{~min}$. When the testing session started, the mouse 


\section{A}
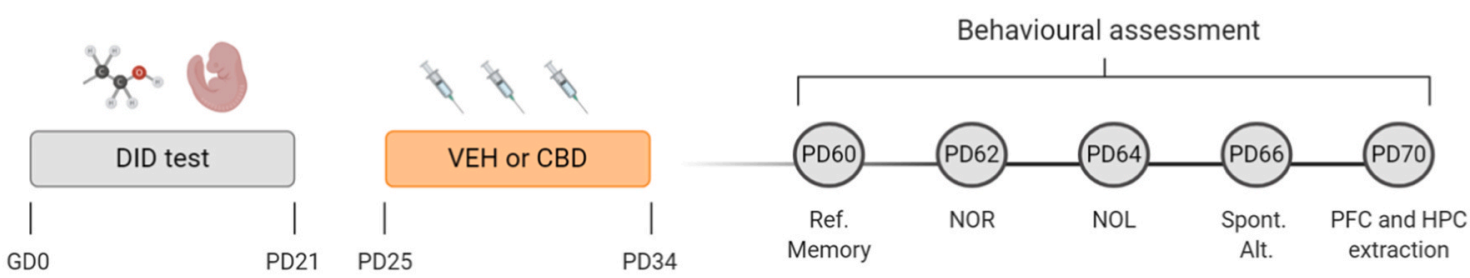

B

Alcohol drinking
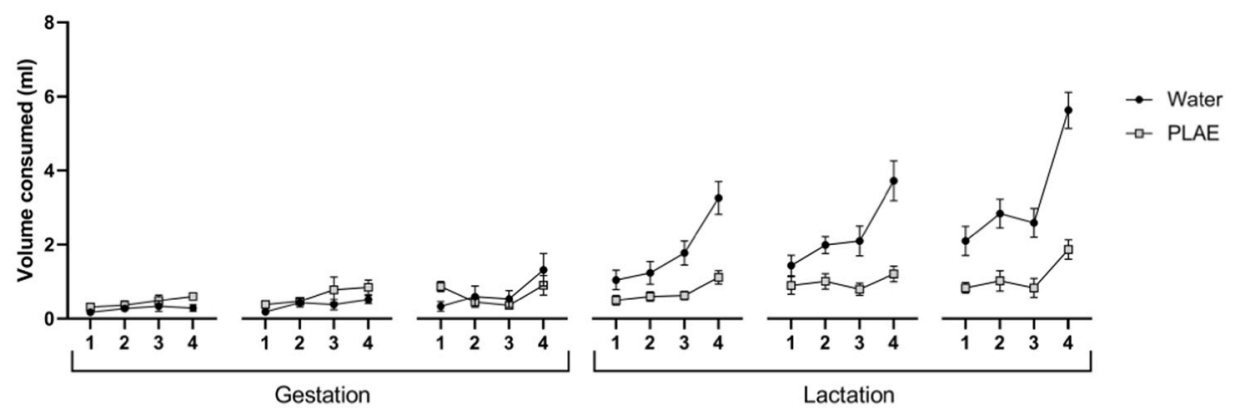

\section{C}

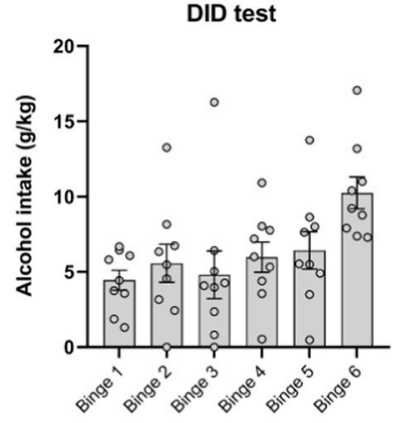

Fig. 1. Experimental design and alcohol consumption by dams during binge-like drinking sessions. (A) Schematic representation of experimental design extensively described above. (B) Volumes (ml) of water or alcohol drinking by pregnant mice during DID test. (C) Alcohol intake ( $\mathrm{g}$ of alcohol per kg of body weight) in the six binge-like drinking sessions throughout gestational and lactation periods ( $\mathrm{n}=9$ dams). Data are presented as mean \pm SEM. CBD, cannabidiol; DID, drinking-in-dark test; HPC, hippocampus; NOL, novel object location; NOR, novel object recognition; PFC, prefrontal cortex; Ref. memory, reference memory; VEH, vehicle; Spont. Alt, spontaneous alternation.

was placed back into the maze with the blockage removed and was allowed to freely explore the three arms for $5 \mathrm{~min}$. One visual clue was placed on the furthest wall of each arm in order to help the mouse recognise the arms of the maze. The time spent in each of the three arms was measured by the Smart Software (Panlab s.l.u., Barcelona, Spain). The preference ratio was calculated by: $\frac{t \text { novel arm }}{t \text { total }}$, being " $t$ " the time each mouse spent exploring the arms.

\subsection{Novel Object Recognition (NOR) task}

$48 \mathrm{~h}$ following the reference memory test, this task was performed as described previously [32] with minor modifications. Black open boxes $(24 \mathrm{~cm} \times 24 \mathrm{~cm} \times 15 \mathrm{~cm})$ and plastic toys of similar size to mice were used in this experiment. Briefly, the procedure consisted of three phases: habituation, training and test session, always under dim light intensity (30lux). During the habituation, mice were individually acclimatised to the box for $10 \mathrm{~min}$ without objects. After $2 \mathrm{~h}$, the training session took place, and the mice were allowed to explore the box in the presence of two identical objects (familiar objects) for $10 \mathrm{~min}$. Finally, the test session was performed $4 \mathrm{~h}$ after the training. In the test session, a familiar object was replaced by a novel one and the animals were allowed to explore for $10 \mathrm{~min}$. The familiar and novel objects were counterbalanced. The discrimination index was calculated as: $\frac{(t \text { novel }-t \text { familiar })}{(t \text { novel } t \text { familiar })} \quad 100$, being " $t$ " the time a mouse spent exploring the objects (manually recorded from a video by an observer who was blind to the experimental groups).

\subsection{Novel Object Location (NOL) task}

This task was performed $48 \mathrm{~h}$ after NOR. We used black open boxes $(24 \mathrm{~cm} \times 24 \mathrm{~cm} \times 15 \mathrm{~cm})$ and two identical plastic toys, which were different from the ones used in the NOR task. The procedure consisted of the identic three phases as in the NOR task, under the same timing and lighting conditions. However, during the test session, one of the familiar objects was displaced to a new location on the arena. This change of spatial configuration triggers an increase level of exploration compared 
with the non-displaced object, as other authors have previously reported $[33,34]$. Here, the displaced and non-displaced objects were counterbalanced. In this case, the discrimination index was calculated as: $\frac{(t \text { displaced }-t \text { non-displaced })}{(t \text { displaced }+t \text { non-displaced })} x \quad 100$, being " $t$ " the time a mouse spent exploring the objects (manually recorded from a video by an observer who was blind to the experimental groups).

\subsection{Delayed Spontaneous Alternation Task}

This task is commonly used to assess spatial working memory [35, 36]. The same Y-maze as in the section " $2.5^{\prime \prime}$ was used to perform this task. Briefly, each mouse carried out 10 trials over one day, with inter-trial intervals of $30 \mathrm{~min}$. Each trial was composed by two phases: the forced-choice and the free-choice phases, with an intra-trial delay of $40 \mathrm{~s}$. First, in the forced-choice phase, mice were placed in the start arm of the Y-maze that had randomly one arm closed off, so they were forced to choose the other arm. Once the animal was completely inside the arm, it was closed for freely exploration for $10 \mathrm{~s}$, and then removed from the maze. In the free-choice phase, just $40 \mathrm{~s}$ later, the animal was placed again in the start arm and it was allowed to freely choose the arm for exploration. A correct alternation was considered when the animal chose the non-forced arm in the free-choice phase. Then, the percentage of correct alternations was calculated considering 10 trials per mouse.

\subsection{Western blotting assay}

At PD70, animals were euthanized by cervical dislocation and PFC and HPC were dissected and stored at $-80^{\circ} \mathrm{C}$. The tissue was homogenised in lysis buffer [0.15 M NaCl, 1\% TX-100, 10\% glycerol, $1 \mathrm{mM}$ EDTA, $50 \mathrm{mM}$ TRIS $\mathrm{pH}=7.4$ and a phosphatase and protease inhibitor cocktail (Roche, Basel, Switzerland)], using $25 \mu \mathrm{l}$ per mg of tissue. Homogenates were centrifuged at $1000 \mathrm{~g}$ for $20 \mathrm{~min}$ at $4{ }^{\circ} \mathrm{C}$, and the resulting supernatants were collected for protein quantification. The lysate protein concentration was determined using a stock solution of $5 \mathrm{mg} / \mathrm{ml} \mathrm{BSA}$ as a protein standard. Equal amount of protein $(30 \mu \mathrm{g})$ for each sample were mixed with loading buffer (153 mM TRIS $\mathrm{pH}=6.8$, 7.5\% SDS, 40\% glycerol, 5 mM EDTA, 12.5\% 2- $\beta$-mercaptoethanol, and $0.025 \%$ bromophenol blue), loaded onto $10 \%$ polyacrylamide gels, and then transferred to PVDF membranes (Immobilion-P, MERCK, Burlington, USA). Membranes were blocked for 30 min with $5 \%$ bovine serum albumin at RT and then immunoblotted overnight at $4{ }^{\circ} \mathrm{C}$ using primary antibodies (see Table 1). On the next day, membranes were 3 times washed for $5 \mathrm{~min}$ each with T-TBS $1 \mathrm{X}$ [Tris-buffered saline (100 mmol/L $\mathrm{NaCl}, 10 \mathrm{mmol} / \mathrm{L}$ Tris, $\mathrm{pH}=7.4$ ) and $0.1 \%$ Tris-buffered saline Tween20]. Then, membranes were incubated for $1 \mathrm{~h}$ with their respective secondary fluorescent antibodies: anti-mouse (1:2500, IRDye 800, Abcam, Cat\# ab216772, RRID: AB_2857338) and anti-rabbit (1:2500,

Table 1

Primary antibodies.

\begin{tabular}{|c|c|c|c|c|}
\hline Antibody & Description & Host & Dilution & Company \\
\hline $\begin{array}{l}\text { Caspase- } \\
\quad 3\end{array}$ & $\begin{array}{l}\text { Cleaved caspase- } 3 \text { (active } \\
\text { form) }\end{array}$ & Rabbit & $1: 1000$ & Cell Signalling \\
\hline $\mathrm{COX}-2$ & Cyclooxygenase-2 & Rabbit & $1: 1000$ & Cell Signalling \\
\hline IL-6 & Interleukin-6 & Mouse & $1: 1000$ & $\begin{array}{l}\text { Santa Cruz } \\
\text { Biotechnology }\end{array}$ \\
\hline NLRP3 & $\begin{array}{l}\text { NOD-like receptor family, } \\
\text { pyrin domain-containing } \\
\text { protein } 3\end{array}$ & Rabbit & $1: 1000$ & GeneTex \\
\hline $\begin{array}{r}\text { NFkB } \\
\text { p65 }\end{array}$ & $\begin{array}{l}\text { Nuclear factor Kappa-B p65 } \\
\text { subunit (also known as } \\
\text { RelA) }\end{array}$ & Mouse & $1: 500$ & $\begin{array}{l}\text { Santa Cruz } \\
\text { Biotechnology }\end{array}$ \\
\hline $\mathrm{TNF} \alpha$ & $\begin{array}{l}\text { Tumour necrosis factor } \\
\text { alpha }\end{array}$ & Rabbit & $1: 1000$ & Cell Signalling \\
\hline Tubulin & Class III $\beta$-Tubulin & Rabbit & $1: 5000$ & Abcam \\
\hline Tubulin & $\beta$-Tubulin & Mouse & $1: 5000$ & BD Pharmigen \\
\hline
\end{tabular}

DyLight $^{\mathrm{TM}}$ 680, Rockland, Cat\# 611-144-002, RRID: AB_1660962). Protein expression was quantified using an Amersham ${ }^{\mathrm{TM}}$ Typhoon ${ }^{\mathrm{TM}}$ scanner and quantified using Image Studio Lite software v5.2 (LICOR, USA). Protein expression signals were normalised to the detection of housekeeping control protein in the same samples and expressed in terms of fold-change with respect to control values.

\subsection{Data and statistical analyses}

Animals were randomly assigned to an experimental group. During the behavioural manipulations and data interpretation, researchers were blind to the treatment each animal had received. We analysed the results of all the behavioural test and western blotting analyses using two-way ANOVA with factors defined as Group (levels: Water and PLAE) and Treatment (levels: VEH and CBD). When $F$ values achieved significance and there was no significant variance in homogeneity, Bonferroni's post hoc tests were run. Pearson's correlation analysis was used to explore correlations between the variables.

Data are presented as mean \pm SEM. For statistical analyses we used GraphPad Prism 8.0. software. The $\alpha$ level of statistical significance was set at $\mathrm{P}<0.05$. The exact group size for the individual experiments is shown in the corresponding figure legends.

The schematic representation shown in Fig. 1A was created in BioRender.com.

\section{Results}

\subsection{Maternal alcohol consumption}

Dams were allowed to access either water or $20 \%$ alcohol following the 6 weeks of DID schedule explained above. Liquid volumes were recorded throughout the gestation and lactation periods. The water and alcohol consumption (volume in $\mathrm{ml}$ ) over the 6 weeks is presented in Fig. 1B. The fluid intake was then calculated only for dams exposed to alcohol and is represented in Fig. 1C. Our team has previously demonstrated correlation between similar alcohol intakes and moderate-tohigh blood alcohol concentration in dams [10] as well as in pups [27].

\subsection{CBD treatment from PD25 to PD34 improves some cognitive deficits induced by PLAE}

The four behavioural tasks were focused on the cognitive domain, specifically on memory functions.

\subsubsection{CBD counteracts the PLAE-induced effects on reference memory}

The two-way ANOVA reveals a significant interaction effect between group $\mathrm{x}$ treatment factors $\left(\mathrm{F}_{(1,45)}=4.906, \mathrm{p}<0.05\right)$. Subsequent Bonferroni's post-hoc analysis displays a tendency $(\mathrm{p}=0.06)$ to impair reference memory when comparing PLAE-VEH to the control group Water-VEH. Still, PLAE-CBD group showed significantly higher preference for the novel arm as compared to PLAE-VEH $(p<0.05)$, revealing that CBD treatment hampers the detrimental effect on reference memory caused by PLAE (Fig. 2A).

\subsubsection{CBD does not affect the recognition memory}

Two-way ANOVA analysis of the percentage of discrimination index between novel and familiar object exhibits group effect $\left(\mathrm{F}_{(1,46)}=19.98\right.$, $\mathrm{p}<0.001$ ), without treatment or interaction effect (Fig. 2B). Clearly, the PLAE mice showed a lower discrimination index, indicating an impaired recognition memory, regardless of the treatment.

\subsubsection{CBD ameliorates the PLAE-induced dysfunction in object location memory}

The two-way ANOVA reveals a group $\left(\mathrm{F}_{(1,46)}=6.738, \mathrm{p}<0.05\right)$ and interaction effect between group $x$ treatment factors $\left(\mathrm{F}_{(1,46)}=7.598\right.$, $\mathrm{p}<0.01$ ). Bonferroni's multiple comparisons unveil that PLAE-VEH 
A

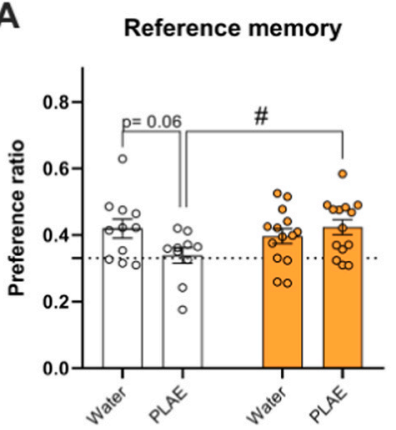

B

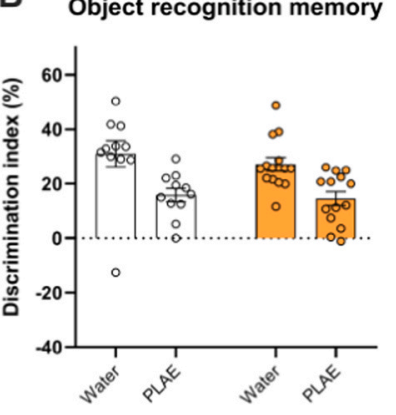

C

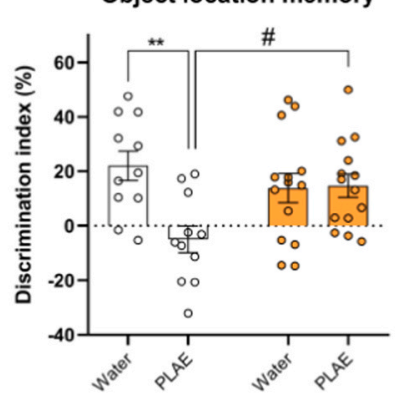

D Spatial Delayed Alternation

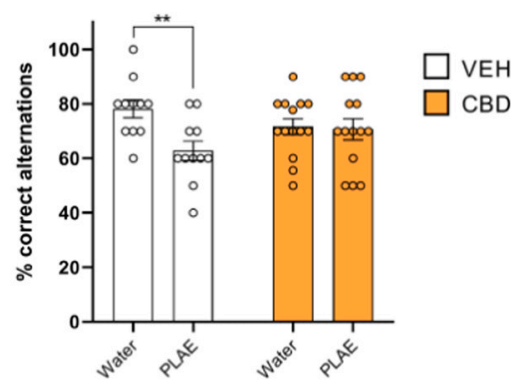

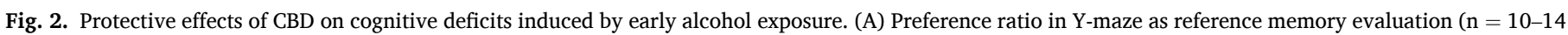

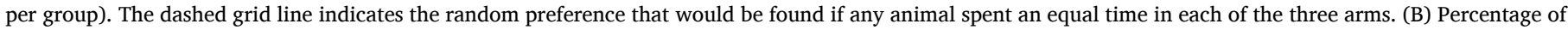

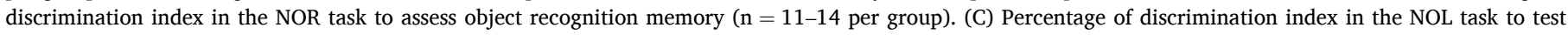

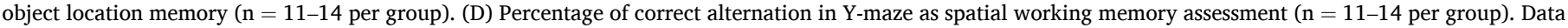

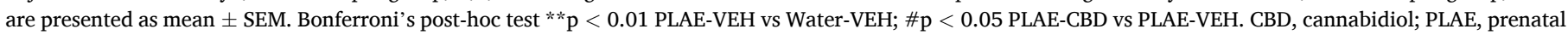
and lactation alcohol exposure; VEH, vehicle.

group exhibited significantly lower discrimination index compared to Water-VEH mice $(\mathrm{p}<0.01)$. In addition, PLAE-CBD animals performed more efficiently the task compared to PLAE-VEH ( $\mathrm{p}<0.05)$, showing greater discrimination index (Fig. 2C). Again, revealing that CBD treatment impedes the deleterious PLAE-induced effect on object location memory.

\subsubsection{CBD faintly hinders spatial working memory deficits induced by PLAE}

Two-way ANOVA analysis displays a group $\left(\mathrm{F}_{(1,46)}=5.605\right.$, $\mathrm{p}<0.05)$ and interaction $\left(\mathrm{F}_{(1,46)}=4.379, \mathrm{p}<0.05\right)$ effects on the percentage of correct alternations in the spatial delayed alternation task (Fig. 2D). Subsequent Bonferroni's post-hoc analyses unveil that PLAEVEH mice carried out lower correct alternations than Water-VEH group ( $\mathrm{p}<0.01$ ). Additionally, the PLAE-CBD group was not significantly different as compared to PLAE-VEH, but neither to Water-VEH. Thus, this result supports that CBD is able to partially prevent spatial working memory deficits caused by PLAE.

\section{3. $C B D$ exerts slight effects on long-term neuroinflammatory response induced by PLAE}

Two-way ANOVA analysis of PFC reveals a group effect for TNF $\alpha$ $\left(\mathrm{F}_{(1,41)}=5.314, \mathrm{p}<0.05\right)$, IL-6 $\left(\mathrm{F}_{(1,39)}=7.987, \mathrm{p}<0.01\right)$, COX-2 $\left(\mathrm{F}_{(1,39)}=4.665, \mathrm{p}<0.05\right)$ and caspase-3 $\left(\mathrm{F}_{(1,40)}=11.29, \mathrm{p}<0.01\right)$, having increased levels of these molecules in PLAE group regardless of treatment (Fig. 3 A-F). A tendency towards an interaction effect between group $x$ treatment factors was found for caspase- $3\left(\mathrm{~F}_{(1,40)}=3.53\right.$, $\mathrm{p}=0.06$ ). Furthermore, we analysed whether the altered inflammatory markers might predict the caspase- 3 elevation, as an indication of detrimental neuroinflammation. Pearson's correlation showed significant positive correlations between levels of caspase- 3 and TNF $\alpha$ $(\mathrm{r}=0.671, \mathrm{p}<0.001)$ and between caspase- 3 and COX-2 $(\mathrm{r}=0.8293$, $\mathrm{p}<0.001$ ). Thus, TNF $\alpha$ and COX-2 in PFC might be useful to predict apoptotic cellular death. However, the Pearson's correlation was not significant for correlation between levels of caspase-3 and IL-6 $(\mathrm{r}=0.2480, \mathrm{p}=0.1046)$ (Fig. $3 \mathrm{G})$.

Two-way ANOVA of HPC unveils a group effect for $\operatorname{TNF} \alpha\left(\mathrm{F}_{(1,41)}\right.$ $=18.35, \mathrm{p}<0.001), \operatorname{NFkB}\left(\mathrm{F}_{(1,39)}=6.375, \mathrm{p}<0.05\right)$ and $\operatorname{COX}-2\left(\mathrm{~F}_{(1,40)}\right.$ $=4.857, \mathrm{p}<0.05)$, showing that PLAE group had higher levels as compared to Water group. Interaction effects were found for $\operatorname{TNF} \alpha\left(\mathrm{F}_{(1,41)}\right.$ $=4.302, \mathrm{p}<0.05)$ and IL-6 $\left(\mathrm{F}_{(1,36)}=4.343, \mathrm{p}<0.05\right)$. Bonferroni's multiple comparisons demonstrate that PLAE-VEH group had greater levels of TNF $\alpha(p<0.001)$ and IL- $6(p<0.05)$ than Water-VEH group. In addition, PLAE-CBD group showed lower levels of TNF $\alpha$ than PLAEVEH group (Fig. 3 H-M), indicating somehow a beneficial effect of
CBD treatment. In case of HPC we did not analyse correlations because the caspase- 3 protein was not modified by PLAE.

\section{Discussion}

In the present study, we demonstrate that CBD administered subchronically throughout a peri-adolescence period can counteract cognitive impairments induced by PLAE in male and female mice. Furthermore, our molecular findings confirm that PLAE long-term neuroinflammatory state in the PFC and HPC can be attenuated by CBD treatment.

The CNS is especially vulnerable to the alcohol effects, primarily throughout developmental periods, like pregnancy and lactation [37]. FASD's most severe manifestations involve behaviour and learning difficulties [3]. In fact, clinical reports have documented marked deficits in high-order functions, such as working memory [38], spatial memory $[39,40]$, and delayed object memory [41]. Our FASD-like mouse model partly resembles the neurobehavioural outcomes reported in those clinical studies, since PLAE animals exhibit dysfunctions on working memory, spatial memory, as well as object recognition and object location memory. We have previously shown toxic blood alcohol levels in dams after the binge-like drinking sessions [10], as well as presence of alcohol in the offspring's blood at two different time points of the lactation (PD15 and PD20) [27], indicating the alcohol exposure throughout lactation.

Although the behavioural consequences of alcohol exposure during gestation and lactation periods are well characterised, the underlying molecular mechanisms are still poorly understood. In this sense, preclinical models provide the opportunity to gain knowledge on those potential mechanisms responsible for FASD. Among the putative mechanisms, it is well described the glia activation following moderateto-high levels of alcohol exposure in rodent models of FASD [6,9]. This activation is accompanied by increased levels of pro-inflammatory cytokines in brain areas, including the cerebral cortex and the HPC $[6,13$, 42], which could remain elevated until the adulthood [10,43]. In line with these findings, we show a long-term neuroinflammatory response within PFC and HPC induced by gestational and lactation alcohol exposure. PLAE mice exhibit higher levels of TNF $\alpha$, IL-6 and COX-2 in both PFC and HPC.

Neuroinflammatory processes could be either neuroprotective or cytotoxic, promoting tissue homoeostasis or enhancing tissue damage [44]. TLR4 is considered a major contributor to innate immune response and a key mediator to activate neuroinflammation following alcohol exposure [12,45]. Moreover, evidence suggests that alcohol-induced apoptotic signalling is mediated by TLR4 $[12,46]$. In addition, prior studies stated cell degeneration in cerebellar vermis while no signal of 


\section{Prefrontal Cortex}
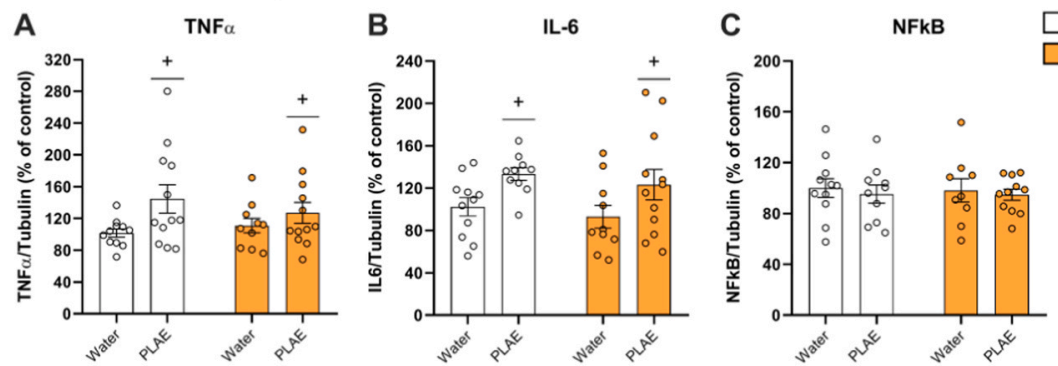

$\square$ VEH
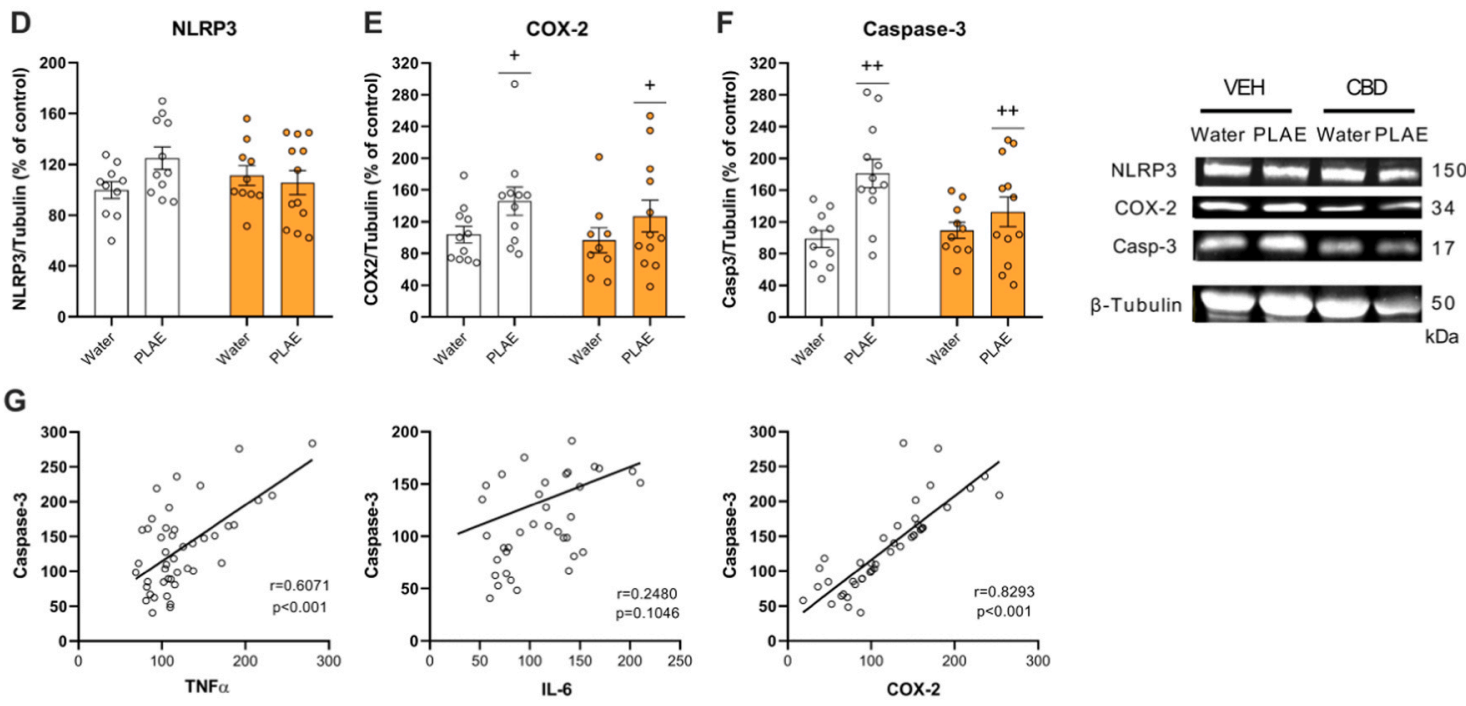

\section{Hippocampus}
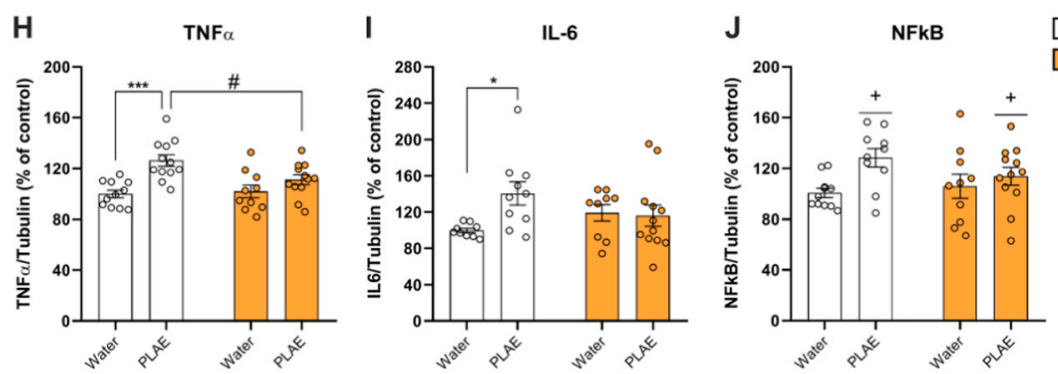

$\square$ VEH
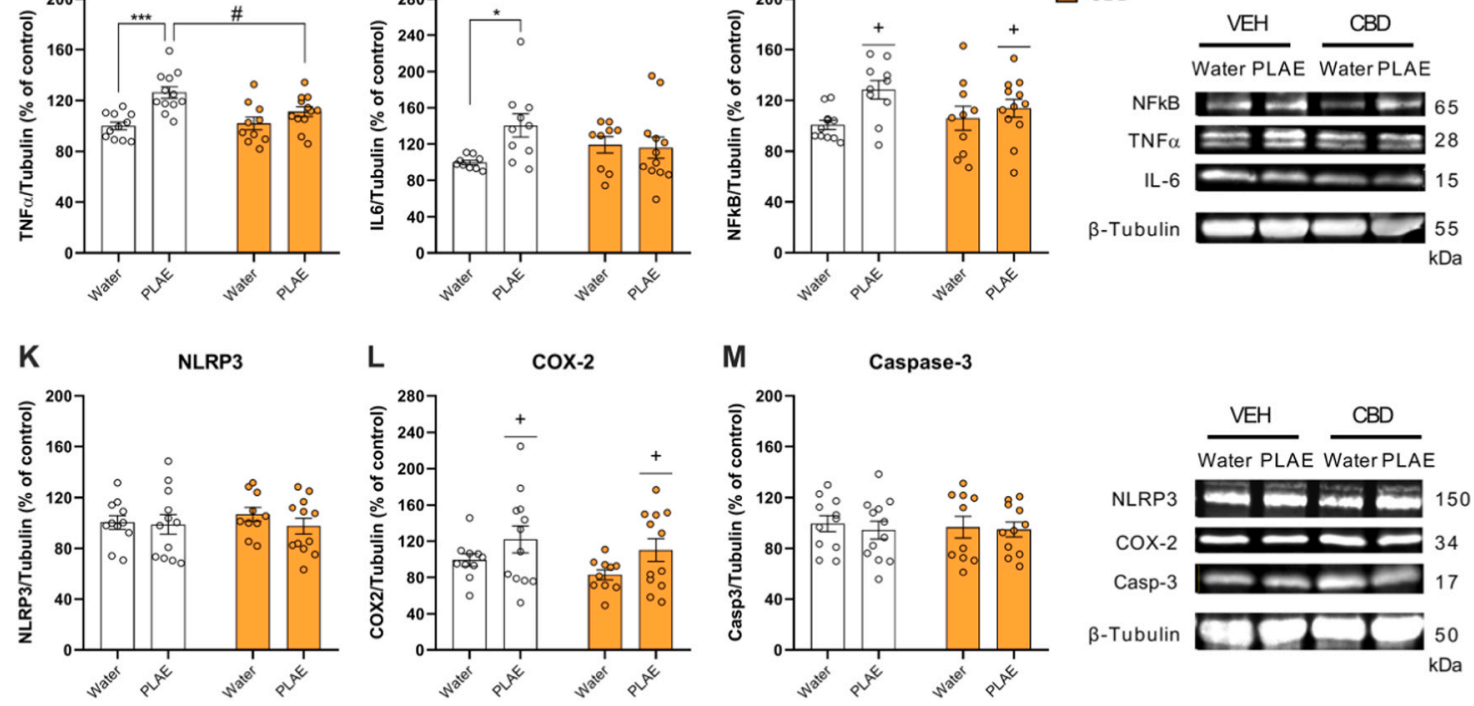

Fig. 3. CBD effect on long-term pro-inflammatory response within prefrontal cortex and hippocampus induced by developmental alcohol exposure. (A-F) Western blot analyses of neuroinflammatory markers (TNF $\alpha$, IL-6, NFkB, NLRP3, COX-2) and apoptotic marker (caspase-3) in PFC ( $n=9-12$ per group). (G) Pearson's correlation between levels of TNF $\alpha$, IL-6 and COX-2 with Caspase-3. (H-M) Western blot analyses of neuroinflammatory and apoptotic markers in HPC ( $\mathrm{n}=9-12$ per group). Data are presented as mean \pm SEM. Group effect of two-way ANOVA test is represented by $+\mathrm{p}<0.05$ and $++\mathrm{p}<0.01$ PLAE vs Water. Bonferroni's post-hoc test *p $<0.05$ and ***p $<0.001$ PLAE-VEH vs Water-VEH; \#p $<0.05$ PLAE-CBD vs PLAE-VEH. CBD, cannabidiol; Casp-3, caspase-3; COX-2, cyclooxygenase-2; IL-6, interleukin-6; NLRP3, NOD-like receptor protein 3; PLAE, prenatal and lactation alcohol exposure; TNF $\alpha$, tumour necrosis factor alpha; VEH, vehicle. 
neuronal loss was found in HPC, despite reporting an increased pro-inflammatory cytokines profile within both regions due to neonatal alcohol exposure [47]. Similarly, we have displayed that PLAE induces long-term brain region-dependent effects on apoptotic signalling, given that caspase-3 levels were higher in PFC, while no changes were found in HPC, as previously reported [10]. Moreover, increased levels of both $\mathrm{TNF} \alpha$ and COX-2 positively correlate to caspase- 3 elevation in PFC, supporting the involvement of pro-inflammatory response in triggering the apoptotic signalling in this brain area. In contrast, PLAE increases NF-kB in HPC without modifications in PFC. Emerging studies support a pivotal role for NF-kB in the pathogenesis of many neurological disorders, either enhancing or mitigating disease [48]. NF-kB mediates neuroinflammatory response and neurodegeneration following excessive and acute alcohol exposure, albeit adaptive changes in NF-kB signalling were suggested to protect brain cells against potent neurotoxicity after repeated cycles of alcohol consumption and withdrawal [49]. In our model, mice were exposed to alcohol repeatedly throughout six weeks, but the brain extractions were carried out after a period of withdrawal. Therefore, the increased levels of NF-kB might enhance anti-apoptotic pathways in HPC and consequently no caspase- 3 deregulations are reported in this area. On the other hand, the pro-apoptotic caspase- 3 elevation in PFC could be due to this region dependent lack of NF-kB activation. However, other concomitant mechanisms occurring within the HPC, like impaired neurogenesis [27] cannot be discarded and should be further evaluated.

The role of neuroinflammation as one of the underlying causes of the behavioural deficits induced by early alcohol exposure is further supported by genetic and pharmacological research. Mice lacking TLR4 do not show behavioural impairments or changes in pro-inflammatory markers following prenatal alcohol exposure indicating that the FASD phenotype is at least partially TLR4 dependent [12]. In the same way, anti-inflammatory drugs like ibuprofen [50] and resveratrol [13] have been proven useful to lessen the FASD phenotype.

Cannabinoids represent an alternative anti-inflammatory strategy to treat several diseases $[14,51]$. Indeed, they might exert positive or negative effects on squeals caused by excessive alcohol use, depending on the cannabinoid compound [15]. Although the detrimental consequences of CB1R agonists on developing brain have been well established [52,53], CBD has recently emerged as promising candidate for several pathological conditions, including alcohol-related harms [54]. Given that preliminary clinical data has already shed light on the positive CBD effects on FASD [55,56], preclinical evidence is firmly necessary. Here, we demonstrate that CBD treatment during peri-adolescence period can attenuate cognitive deficits induced by PLAE, without modifications in control group. Specifically, reference and object location memories were improved by CBD. An interaction between factors was also found for delayed spatial working memory, showing that CBD might slightly attenuate PLAE-induced working memory defect. On the other hand, $\mathrm{CBD}$ does not rescue recognition memory deficits. Therefore, the main positive effect of CBD's is on spatial memory. Similarly, other studies conducted to evaluate neurological disorders proposed that CBD shows therapeutic benefit for spatial and working memory deficits induced by hepatic encephalopathy [57], hypoxic brain injury [58], as well as in preclinical models of infection-induced inflammatory disorders like polyinosinic-polycytidilic acid infection [59] or cerebral malaria [20]. Notwithstanding, other reports propose a dose-dependent CBD effect on recognition memory improvement. For instance, higher doses of CBD counteracted recognition memory impairment induced by a pharmacological model of schizophrenia, whereas lower doses did not [60]. Overall, CBD administration appears to improve cognitive deficits in several domains, which may vary depending on the pathological condition and/or the dose of CBD.

Attenuation of pro-inflammatory signalling following CBD administration was associated with cognitive improvements in several preclinical models $[58,61]$. In the present study, we observe a clear long-term reduction of hippocampal TNF $\alpha$ in PLAE mice treated with CBD.
Down-regulated expression of hippocampal TNF $\alpha$ and/or its receptor 1 has been associated with improved spatial and working memory after CBD treatment [57,62]. Conversely, another study has associated CBD-induced improvement of associative learning with a down-regulation of TNF $\alpha$ expression in frontal cortex, but not in HPC [63]. Moreover, we exhibit a slight CBD effect to counteract IL-6 within the HPC. On the opposite, our findings show no long-term CBD anti-inflammatory effects on PFC. Hence, CBD might be exerting brain region-dependent long-lasting anti-inflammatory mechanisms. Previously, others have displayed that CBD-induced anti-inflammatory response might be region dependent [63]. Besides, CBD showed effective neuroprotection in the HPC at $30 \mathrm{mg} / \mathrm{kg}$ dose, while no significant results were demonstrated at $3 \mathrm{mg} / \mathrm{kg}$ in a mouse model of brain ischaemia [58]. In this sense, future research focusing on elucidating the brain-region specific long-lasting inflammatory modulation induced by CBD would be crucial to ameliorate FASD phenotype. However, even though anti-inflammatory mechanism seems to be relevant, other complementary mechanisms exerted by CBD cannot be discarded. Antioxidant properties of $\mathrm{CBD}$ have been proposed as a potential therapeutic strategy for many diseases associated with oxidative stress [64]. A great amount of evidence shows the contribution of reactive oxygen species and other oxidant markers to the CNS damage caused by early alcohol exposure [65]. Furthermore, some natural antioxidant molecules, like epigallocatechin-3-gallate from green tea, mitigate the deleterious effects of prenatal alcohol exposure [66]. Thus, CBD's antioxidant effects might contribute to the beneficial effects shown in this study.

Overall, CBD appears to be a promising therapeutic drug since it could hamper cognitive impairments caused by several pathological conditions, potentially through neuroimmune modulation. In regards to FASD, this is the first preclinical study exhibiting the beneficial effects of CBD on PLAE-induced cognitive deficits, along with long-lasting reduction of pro-inflammatory cytokines, specifically within HPC. Thus, this study provides new insights into the role of non-psychotropic cannabinoids as pharmacological strategy to counteract alterations in cognitive performance of FASD. However, further studies evaluating the dosage-dependent positive or adverse effects of CBD would be necessary to finally elucidate the most effective period and conditions to administer CBD.

\section{Funding}

This study was supported by the Ministerio de Economia y Competitividad (grant number PID2019-104077RB-100), Ministerio de Sanidad (Retic-ISCIII, RD16/017/010 and Plan Nacional sobre Drogas 2018/007). A.G-B received a FI-AGAUR grant from the Generalitat de Catalunya (2019FI_B0081). The Department of Experimental and Health Sciences (UPF) is a "Unidad de Excelencia María de Maeztu" funded by the AEI (CEX2018-000792-M).

\section{Authors contributions}

Alba Garcia-Baos and Olga Valverde were responsible for the study concept and design. Alba Garcia-Baos and Xavi Puig-Reyne carried out the experimental studies. Alba Garcia-Baos, Olga Valverde and Óscar García-Algar participated in the interpretation of findings. Alba GarciaBaos and Olga Valverde drafted the manuscript. All authors critically reviewed the content and approved the final version for publication.

\section{Conflict of interest statement}

The authors declare no conflicts of interest.

\section{Acknowledgements}

The authors wish to thank Laia Alegre Zurano for manuscript 
proofreading. We also thank Phytoplant Research S.L. for CBD supply.

\section{Appendix A. Supporting information}

Supplementary data associated with this article can be found in the online version at doi:10.1016/j.biopha.2021.111813.

\section{References}

[1] C.H. Denny, C.S. Acero, T.S. Naimi, S.Y. Kim, Consumption of alcohol beverages and binge drinking among pregnant women aged 18-44 years - United States, 2015-2017, MMWR Morb. Mortal. Wkly. Rep. 68 (2019) 365-368, https://doi. org/10.15585/mmwr.mm6816a1.

[2] S.E. Maier, J.R. West, Drinking patterns and alcohol-related birth defects, Alcohol Res. Heal 25 (2001) 168-174. /pmc/articles/PMC6707176/?report=abstract (accessed January 15, 2021).

[3] L.F. Wilhoit, D.A. Scott, B.A. Simecka, Fetal alcohol spectrum disorders: characteristics, complications, and treatment, Community Ment. Health J. 53 (2017) 711-718, https://doi.org/10.1007/s10597-017-0104-0.

[4] Basics about FASDs | CDC, (n.d.). 〈https://www.cdc.gov/ncbddd/fasd/facts.html) (accessed 15 January 2021).

[5] C.J.M. Kane, K.D. Phelan, P.D. Drew, Neuroimmune mechanisms in fetal alcohol spectrum disorder, Dev. Neurobiol. 72 (2012) 1302-1316, https://doi.org/ 10.1002/DNEU.22035.

[6] M. Komada, N. Hara, S. Kawachi, K. Kawachi, N. Kagawa, T. Nagao, Y. Ikeda, Mechanisms underlying neuro-inflammation and neurodevelopmental toxicity in the mouse neocortex following prenatal exposure to ethanol, Sci. Rep. 7 (2017) 4934, https://doi.org/10.1038/s41598-017-04289-1.

[7] P.D. Drew, C.J.M. Kane, Fetal alcohol spectrum disorders and neuroimmune changes, Int. Rev. Neurobiol. 118 (2014) 41-80, https://doi.org/10.1016/B978-0 12-801284-0.00003-8.

[8] T.S. Bodnar, L.A. Hill, J. Weinberg, Evidence for an immune signature of prenatal alcohol exposure in female rats, Brain Behav. Immun. 58 (2016) 130-141, https:// doi.org/10.1016/j.bbi.2016.05.022.

[9] L.S. Terasaki, J.M. Schwarz, Effects of moderate prenatal alcohol exposure during early gestation in rats on inflammation across the maternal-fetal-immune interface and later-life immune function in the offspring, J. Neuroimmune Pharmacol. 11 (2016) 680-692, https://doi.org/10.1007/s11481-016-9691-8.

[10] L. Cantacorps, S. Alfonso-Loeches, M. Moscoso-Castro, J. Cuitavi, I. Gracia-Rubio, R. López-Arnau, E. Escubedo, C. Guerri, O. Valverde, Maternal alcohol binge drinking induces persistent neuroinflammation associated with myelin damage and behavioural dysfunctions in offspring mice, Neuropharmacology 123 (2017) 368-384, https://doi.org/10.1016/j.neuropharm.2017.05.034.

[11] H. Kumar, T. Kawai, S. Akira, Toll-like receptors and innate immunity, Biochem. Biophys. Res. Commun. 388 (2009) 621-625, https://doi.org/10.1016/j bbrc. 2009.08.062.

[12] M. Pascual, J. Montesinos, S. Montagud-Romero, J. Forteza, M. Rodríguez-Arias, J. Miñarro, C. Guerri, TLR4 response mediates ethanol-induced neurodevelopment alterations in a model of fetal alcohol spectrum disorders, J. Neuroinflamm. 14 (2017) 145, https://doi.org/10.1186/s12974-017-0918-2.

[13] V. Tiwari, K. Chopra, Resveratrol prevents alcohol-induced cognitive deficits and brain damage by blocking inflammatory signaling and cell death cascade in neonatal rat brain, J. Neurochem. 117 (2011) 678-690, https://doi.org/10.1111/ j.1471-4159.2011.07236.x.

[14] L. Cristino, T. Bisogno, V. Di Marzo, Cannabinoids and the expanded endocannabinoid system in neurological disorders, Nat. Rev. Neurol. 16 (2020) 9-29, https://doi.org/10.1038/s41582-019-0284-Z.

[15] A. García-Baos, L. Alegre-Zurano, L. Cantacorps, A. Martín-Sánchez, O. Valverde, Role of cannabinoids in alcohol-induced neuroinflammation, Prog. NeuroPsychopharmacol. Biol. Psychiatry 104 (2021), https://doi.org/10.1016/j. pnpbp.2020.110054.

[16] T. Bisogno, L. Hanuš, L. De Petrocellis, S. Tchilibon, D.E. Ponde, I. Brandi, A S. Moriello, J.B. Davis, R. Mechoulam, V. Di Marzo, Molecular targets for cannabidiol and its synthetic analogues: effect on vanilloid VR1 receptors and on the cellular uptake and enzymatic hydrolysis of anandamide, Br. J. Pharmacol. 134 (2001) 845-852, https://doi.org/10.1038/sj.bjp.0704327.

[17] M.V. Fogaça, A.C. Campos, L.D. Coelho, R.S. Duman, F.S. Guimarães, The anxiolytic effects of cannabidiol in chronically stressed mice are mediated by the endocannabinoid system: role of neurogenesis and dendritic remodeling, Neuropharmacology 135 (2018) 22-33, https://doi.org/10.1016/j neuropharm.2018.03.001.

[18] S. Burstein, Cannabidiol (CBD) and its analogs: a review of their effects on inflammation, Bioorg. Med. Chem. 23 (2015) 1377-1385, https://doi.org/ 10.1016/j.bmc.2015.01.059.

[19] A.M. Martín-Moreno, D. Reigada, B.G. Ramírez, R. Mechoulam, N. Innamorato A. Cuadrado, M.L. De Ceballos, Cannabidiol and other cannabinoids reduce microglial activation in vitro and in vivo: relevance to alzheimer's disease, Mol. Pharmacol. 79 (2011) 964-973, https://doi.org/10.1124/mol.111.071290.

[20] A.C. Campos, F. Brant, A.S. Miranda, F.S. Machado, A.L. Teixeira, Cannabidiol increases survival and promotes rescue of cognitive function in a murine model of cerebral malaria, Neuroscience 289 (2015) 166-180, https://doi.org/10.1016/j. neuroscience.2014.12.051.
[21] G. Esposito, C. Scuderi, M. Valenza, G.I. Togna, V. Latina, D. de Filippis, M. Cipriano, M.R. Carratù, T. Iuvone, L. Steardo, Cannabidiol reduces A $\beta$-induced neuroinflammation and promotes hippocampal neurogenesis through PPAR involvement, PLoS One 6 (2011), e28668, https://doi.org/10.1371/journal. pone.0028668.

[22] A.B. Sonego, D.S. Prado, G.T. Vale, J.E. Sepulveda-Diaz, T.M. Cunha, C.R. Tirapelli, E.A. Del Bel, R. Raisman-Vozari, F.S. Guimarães, Cannabidiol prevents haloperidolinduced vacuos chewing movements and inflammatory changes in mice via PPAR $\gamma$ receptors, Brain Behav. Immun. 74 (2018) 241-251, https://doi.org/10.1016/j. bbi.2018.09.014.

[23] L. De Petrocellis, A. Ligresti, A.S. Moriello, M. Allarà, T. Bisogno, S. Petrosino, C. G. Stott, V. Di Marzo, Effects of cannabinoids and cannabinoid-enriched Cannabis extracts on TRP channels and endocannabinoid metabolic enzymes, Br. J. Pharmacol. 163 (2011) 1479-1494, https://doi.org/10.1111/j.14765381.2010.01166.x.

[24] S. Hassan, K. Eldeeb, P.J. Millns, A.J. Bennett, S.P.H. Alexander, D.A. Kendall, Cannabidiol enhances microglial phagocytosis via transient receptor potential (TRP) channel activation, Br. J. Pharmacol. 171 (2014) 2426-2439, https://doi. org/10.1111/bph.12615.

[25] C. Hamelink, A. Hampson, D.A. Wink, L.E. Eiden, R.L. Eskay, Comparison of cannabidiol, antioxidants, and diuretics in reversing binge ethanol-induced neurotoxicity, J. Pharmacol. Exp. Ther. 314 (2005) 780-788, https://doi.org/ 10.1124/jpet.105.085779.

[26] D.J. Liput, D.C. Hammell, A.L. Stinchcomb, K. Nixon, Transdermal delivery of cannabidiol attenuates binge alcohol-induced neurodegeneration in a rodent model of an alcohol use disorder, Pharmacol. Biochem. Behav. 111 (2013) 120-127, https://doi.org/10.1016/j.pbb.2013.08.013.

[27] S. Montagud-Romero, L. Cantacorps, O. Valverde, Histone deacetylases inhibitor trichostatin A reverses anxiety-like symptoms and memory impairments induced by maternal binge alcohol drinking in mice, J. Psychopharmacol. 33 (2019) 1573-1587, https://doi.org/10.1177/0269881119857208.

[28] L. Cantacorps, H. González-Pardo, J.L. Arias, O. Valverde, N.M. Conejo, Altered brain functional connectivity and behaviour in a mouse model of maternal alcohol binge-drinking, Prog. Neuro-Psychopharmacol. Biol. Psychiatry 84 (2018) 237-249, https://doi.org/10.1016/J.PNPBP.2018.03.006.

[29] Drinking Levels Defined | National Institute on Alcohol Abuse and Alcoholism (NIAAA), (n.d.). 〈https://www.niaaa.nih.gov/alcohol-health/overview-alcoho l-consumption/moderate-binge-drinking (accessed 19 December 2020).

[30] J.M. Nichols, B.L.F. Kaplan, Immune responses regulated by cannabidiol, Cannabis Cannabinoid Res 5 (2020) 12-31, https://doi.org/10.1089/can.2018.0073.

[31] A.K. Kraeuter, P.C. Guest, Z. Sarnyai, The Y-maze for assessment of spatial working and reference memory in mice, in: Methods Mol. Biol., Humana Press Inc., 2019, pp. 105-111, https://doi.org/10.1007/978-1-4939-8994-2_10.

[32] M. Maccarrone, O. Valverde, M.L. Barbaccia, A. Castañé, R. Maldonado, C. Ledent, M. Parmentier, A. Finazzi-Agrò, Age-related changes of anandamide metabolism in $\mathrm{CB}{ }_{1}$ cannabinoid receptor knockout mice: correlation with behaviour, Eur. J. Neurosci. 15 (2002) 1178-1186, https://doi.org/10.1046/j.14609568.2002.01957.x

[33] J.F. Morici, P. Bekinschtein, N.V. Weisstaub, Medial prefrontal cortex role in recognition memory in rodents, Behav. Brain Res. 292 (2015) 241-251, https:// doi.org/10.1016/j.bbr.2015.06.030.

[34] L.M. DeVito, H. Eichenbaum, Distinct contributions of the hippocampus and medial prefrontal cortex to the "what-where-when" components of episodic-like memory in mice, Behav. Brain Res. 215 (2010) 318-325, https://doi.org/10.1016/ j.bbr.2009.09.014.

[35] R.M.J. Deacon, D.M. Bannerman, B.P. Kirby, A. Croucher, J.N.P. Rawlins, Effects of cytotoxic hippocampal lesions in mice on a cognitive test battery, Behav. Brain Res. 133 (2002) 57-68, https://doi.org/10.1016/S0166-4328(01)00451-X.

[36] R.M.J. Deacon, J.N.P. Rawlins, T-maze alternation in the rodent, Nat. Protoc. 1 (2006) 7-12, https://doi.org/10.1038/nprot.2006.2.

[37] S. Alfonso-Loeches, C. Guerri, Molecular and behavioral aspects of the actions of alcohol on the adult and developing brain, Crit. Rev. Clin. Lab. Sci. 48 (2011) 19-47, https://doi.org/10.3109/10408363.2011.580567.

[38] C. Rasmussen, Executive functioning and working memory in fetal alcohol spectrum disorder, Alcohol. Clin. Exp. Res. 29 (2005) 1359-1367, https://doi.org/ 10.1097/01.alc.0000175040.91007.d0.

[39] K.L. Kaemingk, P.T. Halverson, Spatial memory following prenatal alcohol exposure: more than a material specific memory deficit, Child Neuropsychol. 6 (2000) 115-128, https://doi.org/10.1076/chin.6.2.115.7058.

[40] D.A. Hamilton, P. Kodituwakku, R.J. Sutherland, D.D. Savage, Children with Fetal Alcohol Syndrome are impaired at place learning but not cued-navigation in a virtual Morris water task, Behav. Brain Res. 143 (2003) 85-94, https://doi.org/ 10.1016/S0166-4328(03)00028-7.

[41] A. Uecker, L. Nadel, Spatial locations gone awry: object and spatial memory deficits in children with fetal alcohol syndrome, Neuropsychologia 34 (1996) 209-223, https://doi.org/10.1016/0028-3932(95)00096-8.

[42] P.D. Drew, J.W. Johnson, J.C. Douglas, K.D. Phelan, C.J.M. Kane, Pioglitazone blocks ethanol induction of microglial activation and immune responses in the hippocampus, cerebellum, and cerebral cortex in a mouse model of fetal alcohol spectrum disorders, Alcohol. Clin. Exp. Res. 39 (2015) 445-454, https://doi.org/ 10.1111/acer.12639.

[43] L.G. Chastain, T. Franklin, O. Gangisetty, M.A. Cabrera, S. Mukherjee, P. Shrivastava, S. Jabbar, D.K. Sarkar, Early life alcohol exposure primes hypothalamic microglia to later-life hypersensitivity to immune stress: possible epigenetic mechanism, Neuropsychopharmacology 44 (2019) 1579-1588, https:// doi.org/10.1038/s41386-019-0326-7. 
[44] A. Aguzzi, B.A. Barres, M.L. Bennett, Microglia: scapegoat, saboteur, or something else? Science 339 (2013) 156-161, https://doi.org/10.1126/science.1227901.

[45] S. Fernandez-Lizarbe, M. Pascual, C. Guerri, Critical role of TLR4 response in the activation of microglia induced by ethanol, J. Immunol. 183 (2009) 4733-4744, https://doi.org/10.4049/jimmunol.0803590.

[46] S. Alfonso-Loeches, M. Pascual-Lucas, A.M. Blanco, I. Sanchez-Vera, C. Guerri, Pivotal role of TLR4 receptors in alcohol-induced neuroinflammation and brain damage, J. Neurosci. 30 (2010) 8285-8295, https://doi.org/10.1523/ JNEUROSCI.0976-10.2010.

[47] L.A. Topper, B.C. Baculis, C. Fernando Valenzuela, Exposure of neonatal rats to alcohol has differential effects on neuroinflammation and neuronal survival in the cerebellum and hippocampus, 2012. https://doi.org/10.1186/s12974-015-0382-9.

[48] M.P. Mattson, M.K. Meffert, Roles for NF- $\mathrm{kB}$ in nerve cell survival, plasticity, and disease, Cell Death Differ. 13 (2006) 852-860, https://doi.org/10.1038/sj. cdd.4401837.

[49] T. Yakovleva, I. Bazov, H. Watanabe, K.F. Hauser, G. Bakalkin, Transcriptional control of maladaptive and protective responses in alcoholics: a role of the NF- $\mathrm{BB}$ system, Brain Behav. Immun. 25 (Suppl 1) (2011) 29-38, https://doi.org/10.1016/ j.bbi.2010.12.019.

[50] M.J. Goodfellow, Y.J. Shin, D.H. Lindquist, Mitigation of postnatal ethanol-induced neuroinflammation ameliorates trace fear memory deficits in juvenile rats, Behav. Brain Res. 338 (2018) 28-31, https://doi.org/10.1016/j.bbr.2017.09.047.

[51] L. Walter, N. Stella, Cannabinoids and neuroinflammation, Br. J. Pharmacol. 141 (2004) 775-785, https://doi.org/10.1038/sj.bjp.0705667.

[52] E.W. Fish, L.B. Murdaugh, C. Zhang, K.E. Boschen, O. Boa-Amponsem, H. N. Mendoza-Romero, M. Tarpley, L. Chdid, S. Mukhopadhyay, G.J. Cole, K. P. Williams, S.E. Parnell, Cannabinoids exacerbate alcohol teratogenesis by a CB1hedgehog interaction, Sci. Rep. 9 (2019) 16057, https://doi.org/10.1038/s41598019-52336-w.

[53] H.V. Weimar, H.R. Wright, C.R. Warrick, A.M. Brown, J.M. Lugo, T.G. Freels, R. J. McLaughlin, Long-term effects of maternal cannabis vapor exposure on emotional reactivity, social behavior, and behavioral flexibility in offspring, Neuropharmacology 179 (2020), 108288, https://doi.org/10.1016/j. neuropharm.2020.108288.

[54] J. Turna, S.K. Syan, B.N. Frey, B. Rush, M.J. Costello, M. Weiss, J. MacKillop, Cannabidiol as a novel candidate alcohol use disorder pharmacotherapy: a systematic review, Alcohol. Clin. Exp. Res. 43 (2019) 550-563, https://doi.org/ 10.1111/acer.13964.

[55] G. Koren, R. Cohen, O. Sachs, Use of cannabis in fetal alcohol spectrum disorder, Cannabis Cannabinoid Res. (2020), https://doi.org/10.1089/can.2019.0056.

[56] M. Dor, G. Koren, R. Cohen, M.B.A. Pharm, Open access rambam maimonides medical journal special issue on cannabis in medicine with guest editor medicinal use of cannabis in children and pregnant women, Rambam Maimonides Med. J. 11 (2020), https://doi.org/10.5041/RMMJ.10382.

[57] I. Magen, Y. Avraham, Z. Ackerman, L. Vorobiev, R. Mechoulam, E.M. Berry, Cannabidiol ameliorates cognitive and motor impairments in mice with bile duct ligation, J. Hepatol. 51 (2009) 528-534, https://doi.org/10.1016/j. jhep.2009.04.021.

[58] A.P. Schiavon, L.M. Soares, J.M. Bonato, H. Milani, F.S. Guimarães, R.M. Weffort de Oliveira, Protective effects of cannabidiol against hippocampal cell death and cognitive impairment induced by bilateral common carotid artery occlusion in mice, Neurotox. Res. 26 (2014) 307-316, https://doi.org/10.1007/s12640-0149457-0.

[59] A.L. Osborne, N. Solowij, I. Babic, X.F. Huang, K. Weston-Green, Improved social interaction, recognition and working memory with cannabidiol treatment in a prenatal infection (poly I:C) rat model, Neuropsychopharmacology 42 (2017) 1447-1457, https://doi.org/10.1038/npp.2017.40.

[60] F.V. Gomes, R. Llorente, E.A. Del Bel, M.P. Viveros, M. López-Gallardo, F. S. Guimarães, Decreased glial reactivity could be involved in the antipsychotic-like effect of cannabidiol, Schizophr. Res. 164 (2015) 155-163, https://doi.org/ 10.1016/j.schres.2015.01.015.

[61] A. María Martín-Moreno, D. Reigada, B.G. Ramírez, R. Mechoulam, N. Innamorato, A. Cuadrado, M.L. de Ceballos, Cannabidiol and Other Cannabinoids Reduce Microglial Activation In Vitro and In Vivo: Relevance to Alzheimer's Disease, (2011). https://doi.org/10.1124/mol.111.071290.

[62] I. Magen, Y. Avraham, Z. Ackerman, L. Vorobiev, R. Mechoulam, E.M. Berry, Cannabidiol ameliorates cognitive and motor impairments in bile-duct ligated mice via 5-HT 1A receptor activation, Br. J. Pharmacol. 159 (2010) 950-957, https:// doi.org/10.1111/j.1476-5381.2009.00589.x.

[63] T. Barichello, R.A. Ceretta, J.S. Generoso, A.P. Moreira, L.R. Simões, C.M. Comim, J. Quevedo, M.C. Vilela, A.W. Zuardi, J.A. Crippa, A.L. Teixeira, Cannabidiol reduces host immune response and prevents cognitive impairments in Wistar rats submitted to pneumococcal meningitis, Eur. J. Pharmacol. 697 (2012) 158-164, https://doi.org/10.1016/j.ejphar.2012.09.053.

[64] S. Atalay, I. Jarocka-Karpowicz, E. Skrzydlewska, antioxidants Antioxidative and Anti-Inflammatory Properties of Cannabidiol, (n.d.). 〈https://doi.org/10.3390/ant iox9010021).

[65] Y. Zhang, H. Wang, Y. Li, Y. Peng, A review of interventions against fetal alcohol spectrum disorder targeting oxidative stress, Int. J. Dev. Neurosci. 71 (2018) 140-145, https://doi.org/10.1016/j.ijdevneu.2018.09.001.

[66] L. Almeida-Toledano, V. Andreu-Fernández, R. Aras-López, Ó. García-Algar, L. Martínez, M. Dolores Gómez-Roig, Epigallocatechin gallate ameliorates the effects of prenatal alcohol exposure in a fetal alcohol spectrum disorder-like mouse model, Int. J. Mol. Sci. 22 (2021) (2021) 715, https://doi.org/10.3390/ ijms22020715. 